\title{
O EFEITO DA GRANULOMETRIA NA DECREPITAÇÃO DURANTE A DECOMPOSIÇÃO TÉRMICA DE CALCÁRIOS E CARVÃo
}

\author{
Paula Manoel Crnkovic* e Wagner Luiz Polito \\ Instituto de Química de São Carlos, Universidade de São Paulo, Av. do Trabalhador Sancarlense, 400, 13560-970 São Carlos - SP \\ Claudionor Gomes da Silva Filho e Fernando Eduardo Milioli \\ Departamento de Engenharia Mecânica, Universidade de São Paulo, Av. do Trabalhador Sancarlense, 400, 13566-590 São Carlos - SP \\ Josmar Davilson Pagliuso
}

Departamento de Hidráulica e Saneamento, Universidade de São Paulo, Av. do Trabalhador Sancarlense, 400,

13566-590 São Carlos - SP

Recebido em 21/2/03; aceito em 29/7/03

\begin{abstract}
THE PARTICLE SIZE EFFECT ON DECREPITATION DURING THE THERMAL DECOMPOSITION OF LIMESTONES AND COAL. The use of fluidized bed combustors to burn coal is largely studied to permit the addition of limestone to capture $\mathrm{SO}_{2}$. The particle size for coal and limestone is an important parameter in this process. Thermogravimetry (TG) is used to elucidate the combustion and sulfation processes, but the experimental parameters must be evaluated to be representative in fluidized bed combustors. In the present study the effect of particle size is analyzed in the calcination of limestones and the combustion of coal through the thermogravimetric curve for limestone and derivative thermogravimetric curve for coal. Small peaks representing mass losses between 400 and $500{ }^{\circ} \mathrm{C}$ are observed due to the jumping of particles out of the crucible. This effect, recognized as decrepitation is observed for mid-sized particles provoked by the release of water vapor trapped within their lattice.
\end{abstract}

Keywords: limestone; coal; decrepitation.

\section{INTRODUÇÃO}

A queima de combustíveis fósseis, particularmente o carvão, para a produção de energia é reconhecida como a principal fonte de dióxido de enxofre $\left(\mathrm{SO}_{2}\right)$ introduzido na atmosfera ${ }^{1}$.

Carvões provenientes das minas brasileiras possuem teores de enxofre que variam de 1,0 a 6,0\%. Conseqüentemente, no processo de queima do carvão há a liberação de óxidos de enxofre $\left(\mathrm{SO}_{\mathrm{x}}\right)$ para a atmosfera, $90 \%$ na forma de dióxido de enxofre $\left(\mathrm{SO}_{2}\right)$ e $10 \%$ na forma de trióxido de enxofre $\left(\mathrm{SO}_{3}\right)^{2,3}$. Estes compostos químicos são reconhecidos como altamente poluentes e como indutores da formação da chuva ácida.

Um progresso importante na queima de carvões foi o desenvolvimento da combustão em leito fluidizado. Pesquisas neste campo estão sendo desenvolvidas há mais de três décadas ${ }^{4}$. Este processo consiste na queima de carvão em um leito de partículas sólidas de pequena granulometria $(0,5-1,5 \mathrm{~mm})$, mantido num estado de turbulência (fluidizado) por meio de um fluxo de ar ascendente levado a uma temperatura conveniente para ocorrer a combustão. O leito encontra-se em contato direto com os tubos com água em que é produzido o vapor e, caracteristicamente, produz um elevado coeficiente de transferência de calor, cerca de dez vezes o dos gases de combustão.

Em combustores de leito fluidizado, a combustão é conduzida a temperaturas mais baixas do que nos demais equipamentos, minimizando a formação de óxidos de nitrogênio ${ }^{5}$. De modo igualmente importante, esta tecnologia permite também a introdução de calcário como agente dessulfurizante, resultando em uma diminuição significativa (até $85 \%$ ) das emissões sulfurosas para a atmosfera ${ }^{6}$. Isto ocorre por meio da sulfatação dos calcários, que são compostos de cálcio que atuam como sorventes do $\mathrm{SO}_{2}$, e são atrativos sob o ponto

*e-mail: paulam@sc.usp.br de vista econômico, devido ao seu baixo custo ${ }^{6}$. A estabilidade dos compostos formados com o enxofre diante das condições reinantes durante a queima do carvão é outro ponto vantajoso deste sorvente.

A captura do enxofre pelo calcário pode ser descrita por duas reações globais ${ }^{7}$ :

$$
\begin{aligned}
& \mathrm{CaCO}_{3} \rightarrow \mathrm{CaO}+\mathrm{CO}_{2} \\
& \mathrm{CaO}+\mathrm{SO}_{2}+1 / 2 \mathrm{O}_{2} \rightarrow \mathrm{CaSO}_{4}
\end{aligned}
$$

A reação de calcinação (endotérmica), representada pela Equação 1, transforma o calcário originalmente mais compacto em um material poroso. A reação de sulfatação (exotérmica) (Equação 2) ocorre posteriormente na superfície do substrato $\mathrm{CaO}$.

O tamanho das partículas do carvão e do calcário utilizados no leito fluidizado é um parâmetro muito importante. Partículas de carvão muito grandes podem levar a um baixo aproveitamento do material combustível quando o carvão é rico em cinzas. No caso do tamanho de partículas dos calcários há, na literatura, observações indicando que com o aumento da granulometria aumenta também a resistência à difusão dos gases para dentro da partícula, através da camada de sulfato de cálcio $\left(\mathrm{CaSO}_{4}\right)$ já formada ${ }^{8}$.

Partículas muito pequenas de calcário e de carvão não são usadas com eficiência em leito fluidizado borbulhante, porque são facilmente arrastadas pelos gases para fora do combustor'.

Os calcários existem basicamente em duas formas distintas: calcários calcíticos (formados majoritariamente por $\mathrm{CaCO}_{3}$ ) e dolomíticos [formados por sal duplo de cálcio e magnésio$\left.\mathrm{CaMg}\left(\mathrm{CO}_{3}\right)_{2}\right]$. Ambas as formas podem ser empregadas com a finalidade de serem sorventes de $\mathrm{SO}_{2}$ durante a combustão de carvão em leito fluidizado ${ }^{9,10}$. As camadas calcárias têm origem na acumulação de organismos inferiores, principalmente de ambiente marinho, e na precipitação do carbonato de cálcio e magnésio dissolvidos nas águas dos rios, lagos, mares e fontes de água mineralizadas ${ }^{11}$. 
O carvão é quimica e fisicamente um mineral heterogêneo. Principalmente, consiste de carbono com pequenas quantidades em massa de enxofre, nitrogênio e hidrogênio. $O$ carvão é uma rocha sedimentar de origem orgânica, formado a partir da vegetação consolidada entre os estratos de rochas alteradas pela combinação dos efeitos da ação microbiana, pressão e calor durante um considerável período de tempo ${ }^{12}$. No carvão, o enxofre encontra-se nas formas orgânicas e inorgânicas. O enxofre orgânico ocorre dentro da estrutura do carvão como tiofenos, tioéteres e mercaptanas. O enxofre inorgânico ocorre como pirita, marcasita e melancovita ${ }^{13}$.

É próprio de matériais geológicos, como os calcários ${ }^{14}$ e carvões, apresentarem o fenômeno da decrepitação quando submetidos à análise térmica. Wendland ${ }^{15}$ mencionou o efeito da decrepitação relacionada com o tamanho das partículas, mas, não fez qualquer referência à faixa granulométrica em que este evento ocorre.

A decrepitação deve-se a mini-explosões dentro das partículas, resultando em ejeções de pequenas quantidades de amostra para fora do suporte. Assim, este fenômeno não faz parte da decomposição térmica das amostras, mas, é sim um fenômeno termomecânico ${ }^{16}$.

Este trabalho tem como objetivo demonstrar pelas curvas TG e DTG quais as dimensões das partículas de calcário e carvão que apresentam decrepitação e como este efeito interfere nos resultados, para que os estudos TG possam ser representativos e aplicáveis em combustores de leito fluidizado.

\section{PARTE EXPERIMENTAL}

Os materiais estudados foram o calcário calcítico procedente de Itaú de Minas (MG), o calcário dolomítico procedente de Piracicaba (SP) e o carvão mineral CE 4500 procedente de Criciúma (SC), todos coletados diretamente das minas. A análise química dos calcários calcítico e dolomítico está indicada na Tabela 1 .

A análise elementar e a análise imediata do carvão estão indicadas na Tabela 2.

A análise imediata por termogravimetria envolve o aquecimento da amostra de carvão com fluxo programado de nitrogênio e oxigênio $^{17}$. Os experimentos foram feitos com massa de $20 \mathrm{mg}$ na sequiência experimental descrita na Tabela 3.

Segundo Warne ${ }^{18}$, a análise imediata é utilizada para caracterizar o carvão para os seguintes parâmetros: perda de água (fornece dados sobre o poder calorífico), voláteis (indica os materiais que queimam acima do leito ou fora da partícula na fase gasosa), carbono fixo (parâmetro que indica a queima no interior do leito ou da partícula) e cinzas (material a ser descartado, indica a eficiência térmica do combustível).

Os materiais de estudo (calcários e carvão) foram obtidos a partir da pedra bruta de mina, submetidos no laboratório à britagem por moinho de martelo e posterior seleção por peneiras vibratórias semiindustriais e laboratoriais.

As granulometrias dos materiais para ensaio foram obtidas sempre por meio de seleção entre duas peneiras sucessivas da série ASTM, de modo a compor a faixa mais estreita possível e minimizar o efeito da distribuição granulométrica. A granulometria média foi calculada por meio da média aritmética da abertura da malha de duas peneiras sucessivas, dentro da faixa escolhida. Estes valores estão indicados na Tabela 4.

As granulometrias médias selecionadas para os calcários foram 44, 115, 390, 462, 545 e 650 um e para o carvão, 44, 115, 390, 775, 1205 e $1840 \mu \mathrm{m}$. A escolha destas faixas granulométricas foi devida à representatividade dentro dos objetivos deste trabalho e também por serem de aplicação prática em combustores de leito fluidizado.

A termobalança usada nos experimentos foi da marca Shimadzu modelo 51H. Tanto para o calcário quanto para o carvão as condições experimentais foram: massa da amostra de $10,0 \pm 0,5 \mathrm{mg}$, razão de aquecimento de $30{ }^{\circ} \mathrm{C} \mathrm{min}{ }^{-1}$ até $850{ }^{\circ} \mathrm{C}$ e patamar de 5 min nesta temperatura. $\mathrm{O}$ gás de arraste foi ar sintético com vazão de $100 \mathrm{~mL}$ $\mathrm{min}^{-1}$ regulado com controlador de fluxo AALBORG modelo G19618C. O suporte da amostra foi de platina com dimensões de 2,5 $\mathrm{mm}$ de profundidade por $5,7 \mathrm{~mm}$ de diâmetro.

Para os calcários foram feitas as microscopias eletrônicas de varredura (MEV), com o aparelho Leo Electon Microscopy 440 (ZEIZ/ LAIKA). Para esta observação, as amostras foram preparadas de acordo com o seguinte procedimento: inicialmente pesaram-se 50,0 $\mathrm{mg}$ de amostras que foram transferidas para um frasco de vidro com capacidade de 20,0 mL. Adicionaram-se $10 \mathrm{~mL}$ de acetona e este frasco permaneceu em banho de ultra-som por 10 min. Com o auxílio de uma pipeta de Pasteur, 2 gotas desta suspensão foram transferidas sobre um suporte de alumínio (Stab) previamente pre-

Tabela 1. Análise química do calcário calcítico de Itaú de Minas (MG) e dolomítico de Piracicaba (SP)

\begin{tabular}{ccc}
\hline & \multicolumn{2}{c}{$\%$} \\
\cline { 2 - 3 } Analitos & calcítico & dolomítico \\
\hline $\mathrm{Ca}$ & 35,34 & 17,07 \\
$\mathrm{Mg}$ & 0,35 & 11,03 \\
$\mathrm{Fe}$ & 2,45 & 0,32 \\
$\mathrm{Al}$ & 0,26 & 0,42 \\
$\mathrm{Sr}$ & 0,14 & 0,08 \\
$\mathrm{Mn}$ & 0,05 & 0,09 \\
$\mathrm{~K}$ & 0,09 & 0,13 \\
$\mathrm{~S}$ & 0,05 & 0,18 \\
\hline
\end{tabular}

Tabela 2. Análises elementar e imediata do carvão mineral CE 4500 - proveniente de Criciúma (SC)

\begin{tabular}{|c|c|c|c|c|c|c|c|c|}
\hline \multicolumn{5}{|c|}{ Análise elementar $1 \%$} & \multicolumn{4}{|c|}{ Análise imediata/\% } \\
\hline $\mathrm{C}$ & $\mathrm{N}$ & $\mathrm{O}$ & $\mathrm{H}$ & S & Água & voláteis & Carbono fixo & cinzas \\
\hline 50,59 & 0,90 & 7,93 & 3,52 & 5,14 & 0,81 & 19,25 & 35,44 & 44,5 \\
\hline
\end{tabular}

Tabela 3. Seqüência experimental em balança termogravimétrica para a análise imediata do carvão mineral

\begin{tabular}{cccc}
\hline $\begin{array}{c}\text { Atmosfera e vazão } \\
\left(\mathrm{mL} \min ^{-1}\right)\end{array}$ & $\begin{array}{c}\text { Razão de aquecimento } \\
\left({ }^{\circ} \mathrm{C} \min ^{-1}\right)\end{array}$ & $\begin{array}{c}\text { Intervalo de temperatura } \\
\left(\Delta \mathrm{T} /{ }^{\circ} \mathrm{C}\right)\end{array}$ & $\begin{array}{c}\text { Tempo de isoterma } \\
(\mathrm{min})\end{array}$ \\
\hline $\mathrm{N}_{2}(70)$ & 30 & $27-110$ & 5 \\
$\mathrm{~N}_{2}(70)$ & 45 & $110-950$ & 3 \\
$\mathrm{~N}_{2}(70)+\mathrm{O}_{2}(30)$ & - & 950 & 12 \\
\hline
\end{tabular}


Tabela 4. Granulometrias médias das partículas

\begin{tabular}{ccc}
\hline Faixa granulométrica & \multicolumn{2}{c}{ Valor médio } \\
$\mu \mathrm{m}$ & $\mu \mathrm{m}$ & Mesh \\
\hline $2110-1570$ & 1840 & 13 \\
$1570-840$ & 1205 & 16 \\
$840-710$ & 775 & 23 \\
$590-710$ & 650 & 28 \\
$500-590$ & 545 & 33 \\
$420-500$ & 462 & 38 \\
$350-420$ & 390 & 43 \\
$105-125$ & 115 & 130 \\
$44-54$ & 44 & 263 \\
\hline
\end{tabular}

parado com uma fita adesiva de carbono. Após a secagem do material, este foi levado à metalização, que foi obtida utilizando-se ouro.

\section{RESULTADOS E DISCUSSÃO}

Por meio das curvas TG do calcário calcítico e da curva DTG do carvão, observaram-se pequenas perdas sucessivas de massa entre as temperaturas de 380 e $550{ }^{\circ} \mathrm{C}$ para algumas granulometrias. Estas perdas de massa são devido à projeção de partículas para fora do suporte da amostra e este efeito é conhecido como decrepitação ${ }^{14,16,19}$. O calcário dolomítico não apresentou tal efeito para nenhum tamanho de partícula.

A Figura 1 mostra as curvas TG do calcário calcítico com diferentes granulometrias. Observa-se que a decrepitação (perda de massa) e a decomposição térmica ocorrem em temperaturas distintas. Notase claramente a decrepitação entre 750 e 1100 s, correspondentes às temperaturas entre 380 e $550{ }^{\circ} \mathrm{C}$. Entretanto isto ocorre somente para as partículas de tamanho intermediário, isto é, aquelas de tamanho médio de 390 e $462 \mu \mathrm{m}$. Tal fenômeno não pode ser atribuído à calcinação, pois, para todas as granulometrias esta etapa do processo (Equação 1) é observada somente a partir de $1300 \mathrm{~s}$, correspondente à $650^{\circ} \mathrm{C}$.

A diferença de comportamento em relação à decrepitação entre ambos os calcários é atribuída à estrutura física. As MEVs indicam que o calcário calcítico (Figura 2) apresenta uma estrutura mais compactada em relação ao calcário dolomítico (Figura 3), que apre-

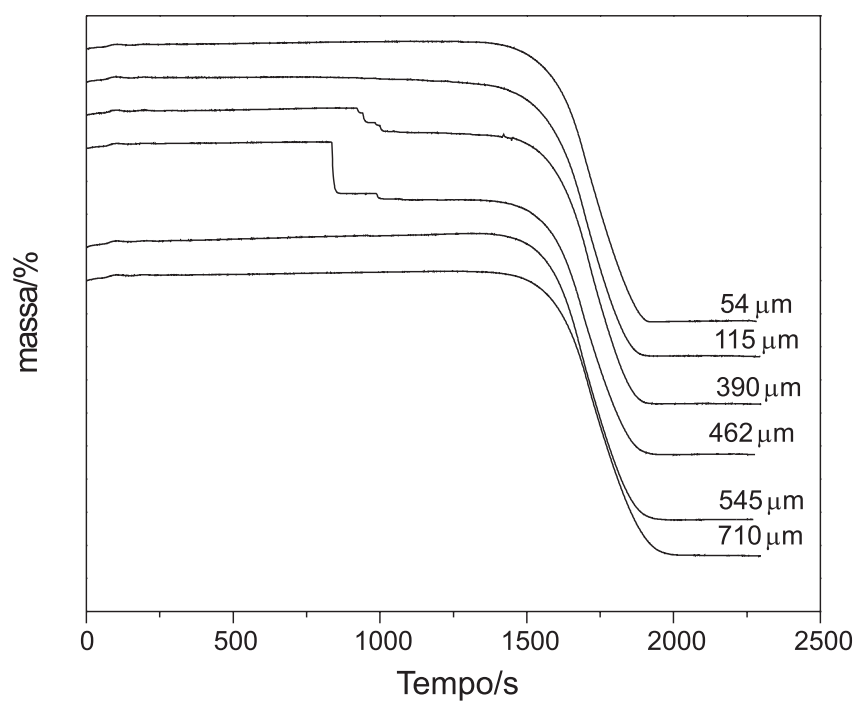

Figura 1. Curvas TG da calcinação do calcário calcítico. Efeito da decrepitação com a granulometria senta uma estrutura lamelar. Como a decrepitação está relacionada com a pressão provocada pela liberação da água dentro do retículo, quando o calcário é mais compactado, a força que este efeito exerce nas paredes é maior, a ponto de provocar a ejeção das partículas para fora do suporte. Para o calcário menos compactado, esta força não é suficientemente grande para provocar tal efeito. Assim, partículas com estrutura física menos compacta estão menos sujeitas à decrepitação.

A Figura 4 mostra as curvas TG e DTG da decomposição térmica do carvão para a granulometria de $335 \mu \mathrm{m}$. Embora não seja possível por meio da curva TG observar claramente a decrepitação e devolatilização como fenômenos em separado, pois os efeitos se sobrepõem, a curva DTG mostra uma série de pequenas oscilações breves, porém, intensas acompanhando o processo da devolatilização. Considera-se que este fato não representa a liberação de diferentes voláteis, pois se assim o fosse, este efeito deveria ocorrer para todas as granulometrias, conseqüentemente, deve ser atribuído à decrepitação.

Na Figura 5 representam-se as curvas DTG para todas as granulometrias de carvão selecionadas para este estudo. Nota-se que a decrepitação é evidenciada para as curvas das granulometrias mé-

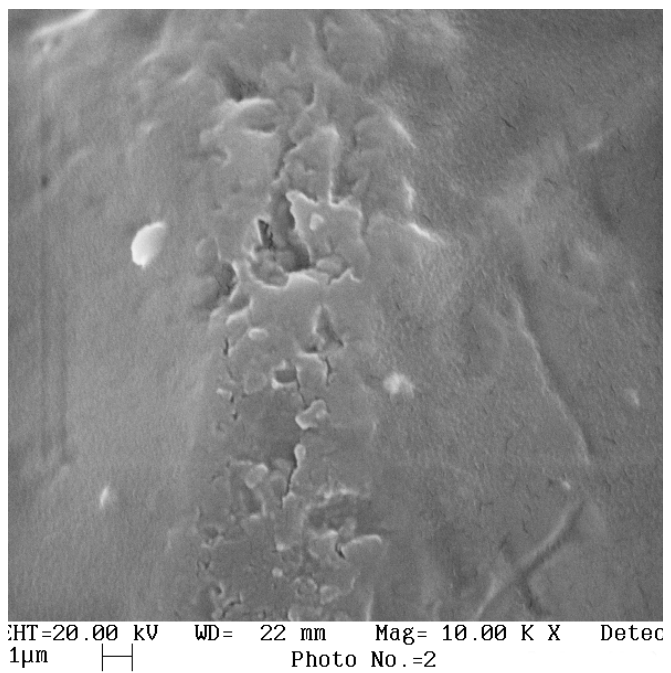

Figura 2. Microscopia eletrônica de varredura (MEV) do calcário calcítico procedente de Itaú de Minas (MG). Amplitude 10.000 vezes

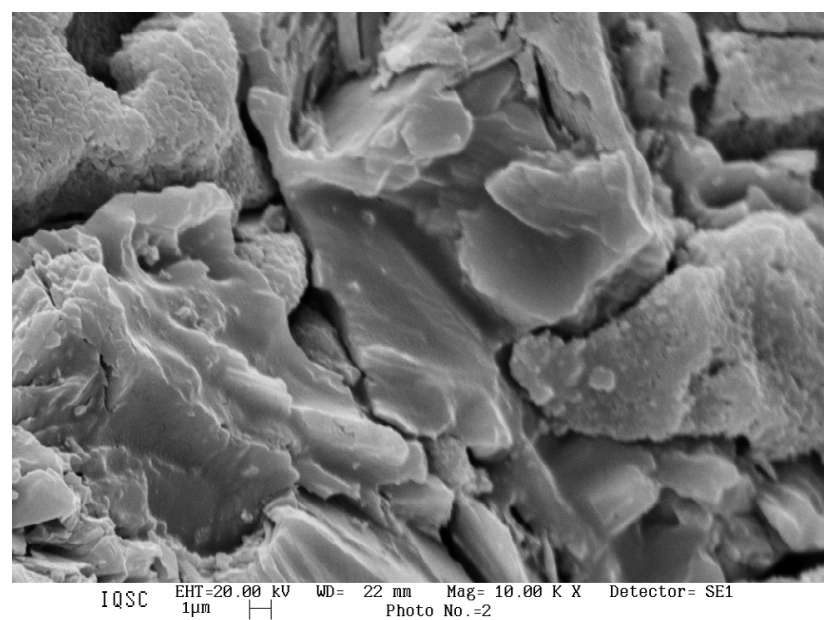

Figura 3. Microscopia eletrônica de varredura (MEV) do calcário dolomítico procedente de Piracicaba (SP). Amplitude 10.000 vezes 


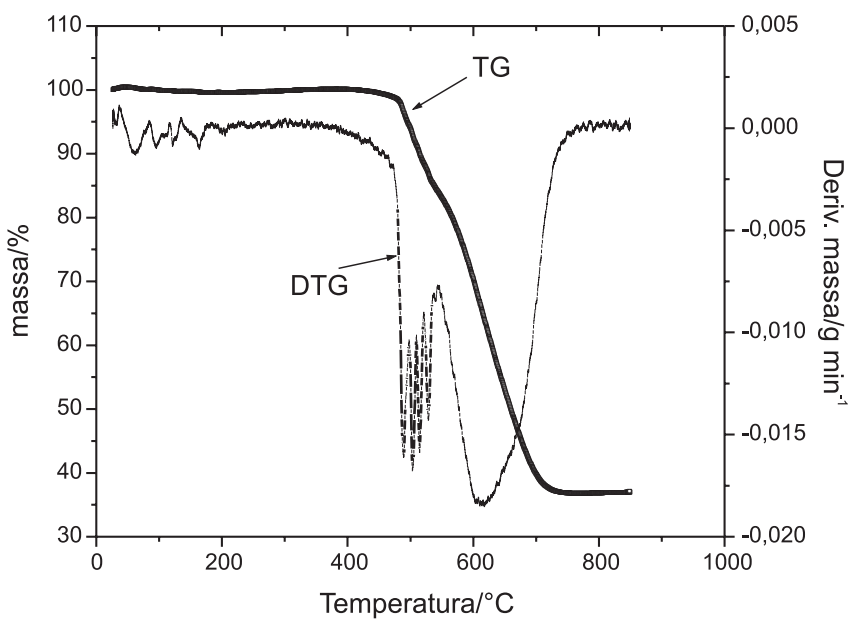

Figura 4. Curvas TG e DTG da decomposição térmica do carvão mineral CE 4500 com granulometria de $335 \mu \mathrm{m}$

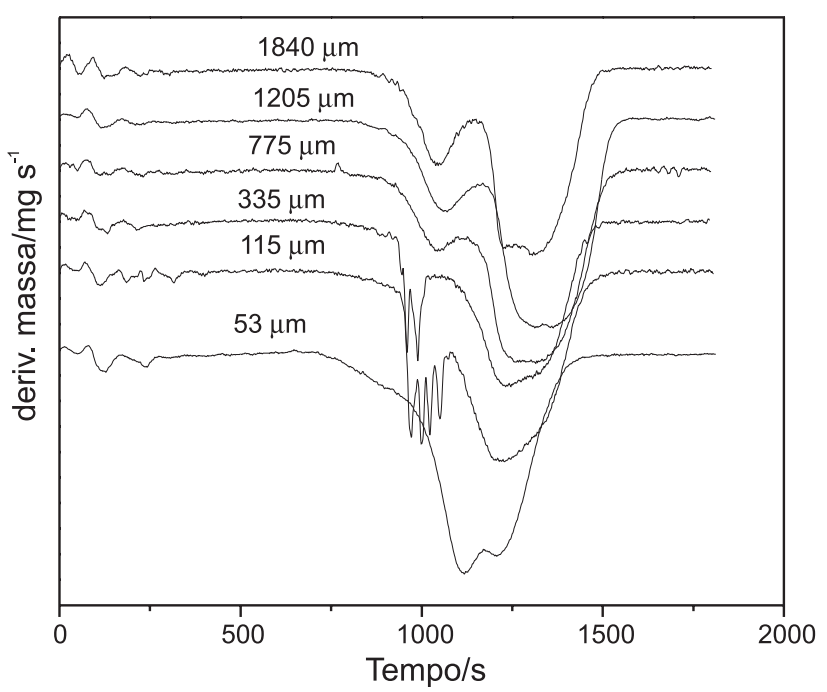

Figura 5. Curvas DTG da decomposição térmica do carvão mineral CE 4500. Efeito da decrepitação com a granulometria

dias de 115 e $325 \mu$ m apenas. Portanto, a melhor forma para se relacionar o efeito do tamanho das partículas de carvão com a decrepitação no processo de sua decomposição térmica é por meio de curvas DTG.

Os resultados apresentados nas Figuras 1, 4 e 5 sugerem que a decrepitação ocorre tanto para o calcário calcítico quanto para o carvão e é melhor relacionada com o tamanho da partícula do que com a decomposição térmica. Desde que o fenômeno é de ejeção das partículas para fora do suporte da amostra, partículas de diferentes tamanhos apresentam comportamentos distintos.

Acredita-se que a decrepitação resulta do aumento da pressão devido à evaporação da água no interior do retículo. As mini-explosões ocorrem quando esta pressão excede a força mecânica das partículas ${ }^{16}$.

As partículas maiores não apresentam decrepitação, possivelmente, porque as mini-explosões não têm potência suficiente para romper a estrutura para vencer sua inércia. Enquanto que, nas partículas menores, por não possuírem massa suficiente, não há competição entre as forças e, conseqüentemente, não são ejetadas para fora do suporte da amostra.

\section{CONCLUSÕES}

Observou-se que a decrepitação ocorre durante a decomposição térmica tanto do calcário de estrutura mais compactada, quanto do carvão. Para ambos, este fenômeno foi observado com as granulometrias de tamanho intermediário, respectivamente, 375 e $443 \mu \mathrm{m}$ para o calcário e 115 e $375 \mu \mathrm{m}$ para o carvão e na mesma faixa de temperaturas, entre 380 e $550{ }^{\circ} \mathrm{C}$.

Devido à grande dificuldade de se prever o comportamento de calcários e carvões em reatores a partir de suas características químicas e físicas, a termogravimetria pode ser um método de análise promissor, pois permite determinar vários parâmetros importantes, como taxa de reação e conversão, que irão determinar em última análise as características operacionais e o projeto do combustor.

Embora os processos no leito e na balança tenham diferenças claras, como por exemplo a razão de aquecimento da partícula e a distribuição de gases em seu envoltório, resultados TG podem ser utilizados, ao menos de maneira semiquantitativa no projeto de reatores.

As condições mais adequadas para que os testes TG tenham aplicabilidade nos reatores são pesquisadas no NETeF (Núcleo de Engenharia Térmica e Fluidos da EESC-USP). Neste trabalho, tratase em particular do fenômeno da decrepitação do calcário e do carvão que é capaz de ejetar partículas para fora do suporte da amostra da balança e, desta forma, produzir informações errôneas para o usuário dos resultados dos testes. Portanto, a granulometria é um parâmetro importante para a adequação dos experimentos termogravimétricos ao se determinar a porcentagem dos voláteis no carvão e a calcinação nos calcários.

\section{AGRADECIMENTOS}

Os autores agradecem à FAPESP pelo projeto regular de pesquisa 99/06055-2, pela bolsa de doutorado 98/14393-2 e ao CNPq pelo projeto integrado de pesquisa 520.563/96-4.

\section{REFERÊNCIAS}

1. Li, Y.; Sadakata, M.; Fuel 1999, 78, 1089.

2. Carello, S. A.; Vilela, A. C. F.; Ind. Eng. Chem. Res. 1993, 32, 3135.

3. Milioli, F. E.; J. Braz. Soc. Mech. Sci. 1996, 2, 127.

4. Lyngfelt, A.; Leckner, B.; Chem. Eng. Sci. 1999, 54, 5573.

5. Wieczorek-Ciurowa, K; Thermochim. Acta 1996, 272, 233.

6. Snyder, R.; Wilson, W. I.; Johnson, I.; Thermochim. Acta 1978, 26, 257.

7. Dam-Johansen, K.; Ostergaard, K.; Chem. Eng. Sci. 1991, 46, 827.

8. Simons, G. A.; Boni, A. R.; AIChE J. 1987, 33, 211.

9. Fuertes, A. B.; Velasco, G.; Fuente, E.; Alvarez, T.; Fuel Process. Technol. 1994, 38, 181 .

10. Yrjas, P.; Iisa, K.; Hupa, M.; Fuel 1995, 74, 395.

11. Malavolta, E.; Manual de química agrícola - adubos e adubação, Ed. Agronômica Ceres: São Paulo, 1981, p. 596.

12. Hessley, R. K.; Reasoner, J. W.; Riley, J. T.; Coal science: an introduction to chemistry technology, and utilization, Wiley: New York, 1986, p. 269.

13. Informativo Anual da Indústria Carbonífera; República Federativa do Brasil, Ministério de Minas e Energia, Departamento Nacional de Produção Mineral 1999.

14. Mc Cauley, R. A.; Johnson, L. A.; Thermochim. Acta 1991, 185, 271.

15. Wendland, W.; Thermal Analysis, Wiley: New York, 1986, p. 814.

16. Santini, M.; Dollimore, D.; Alexander, K.; J. Thermal Anal. 2001, 65, 93.

17. Ernest, C. M.; Fyans, R. L; Perkin-Elmer thermal analysis applications, study, 1981, n. 32, 67.

18. Warne, S. St. J.; Thermochim. Acta 1996, 272, 1.

19. McIntoshi, R. M.; Sharp, J. H.; Wilburn, F. W.; Thermochim. Acta 1990, $165,281$. 